\title{
Neuropsychological abnormalities in AIDS and asymptomatic HIV seropositive patients
}

\author{
G Villa, D Monteleone, C Marra, A Bartoli, A Antinori, F Pallavicini, E Tamburrini, \\ I Izzi
}

\begin{abstract}
Neuropsychological and immunological parameters were studied in 36 AIDS patients with early disease and without clinical, laboratory, and neuroradiological signs of CNS impairment, and also in 33 asymptomatic HIV seropositive subjects. Many AIDS patients performed abnormally on timed psychomotor tasks, tasks involving sequencing and "setshifting", and memory tasks stressing attention, learning, active retrieval, and monitoring of information. Asymptomatic HIV seropositive subjects as a group did not perform significantly worse than controls. However, on the basis of a cut off number of pathological performances on neuropsychological tasks, $52 \cdot 8 \%$ of AIDS and $30.3 \%$ of asymptomatic HIV seropositive subjects had cognitive impairment, compared with $3.9 \%$ of HIV seronegative controls. Low values of CD4+ cells and of CD4 + /CD8 + ratio and high titres of $\mathrm{P}$ 24 antigen in the blood prevailed among subjects with cognitive impairment, especially in the asymptomatic HIV seropositive group.
\end{abstract}

$(\mathcal{F}$ Neurol Neurosurg Psychiatry 1993;56:878-884)

It is the currently accepted notion that HIV has a remarkable neurotropism and that it involves the CNS very early in the course of infection. At the time of seroconversion it can give rise to "aseptic" meningitis, encephalitis, and myelitis. Later, during the stage of asymptomatic seropositivity, it can sustain a persistent CSF infection with cellular and IgG correlates. ${ }^{1}$ Most HIV infected subjects, however, show clinical symptoms, attributable to HIV involvement of the CNS, only in advanced stages of immunodeficiency. More than $40 \%$ of patients with AIDS develop a progressive neurological syndrome, caused by a subacute chronic HIV encephalitis, and referred to as the AIDS dementia complex (ADC), since mental deterioration is its most relevant aspect. ${ }^{2}$ Apart from cases of overt dementia, a high percentage of patients with AIDS and a smaller, but substantial number of patients with AIDS related complex (ARC) exhibit some degree of cognitive impairment for which no opportunistic or other causes can be found. ${ }^{34}$ In a few cases (5-10\%) the appearance of a clinically significant cognitive decline is the event which signals the development of symptomatic immunodeficiency.

The possibility that in early stages of HIV infection there may already be subclinical evidence of cognitive impairment, indicative of evolving damage to CNS, is currently under discussion. Abnormal neuropsychological test results have occasionally been documented in patients with lymphoadenopathy syndrome (LAS), ${ }^{5}$ whereas the existence of some cognitive abnormalities in asymptomatic HIV seropositive individuals has been claimed by some studies ${ }^{36-10}$ and denied by others. ${ }^{11-14}$

As for the neuropsychological profile of this HIV related cognitive impairment, a somewhat surprising conclusion from reviewing the published reports is the large variability in cognitive functioning reported to be affected. Difficulities with complex sequencing, impairment of fine and rapid motor movements, and slowed verbal fluency have been considered as characteristic of ADC. ${ }^{15}$ Alternatively, a selective impairment on tests of psychomotor speed and of visuoconstructive ability, ${ }^{16}$ or a decrease of performance on non-verbal tests of abstraction ${ }^{3}$ have also been stressed. In patients with ARC, a selective impairment on non-verbal memory tasks and on timed tasks of psychomotor speed have been found, ${ }^{17}$ whereas patients with LAS would exhibit deficits in several cognitive areas, including language..$^{5}$ More recently, a significant decrement of psychomotor speed, ${ }^{810}$ verbal memory, 68910 and "frontal"; abilities $^{69}$ has been described in asymptomatic HIV seropositive subjects.

On the basis of these preliminary statements, we embarked upon a systematic neuropsychological study of cognitive abilities in HIV infected individuals in order to obtain: (a) quantification of the HIV related cognitive impairment across the various stages of HIV infection (asymptomatic seropositives, persistent generalised lymphoadenopathy, ARC, AIDS); (b) characterisation of this cognitive impairment, particularly in early stages, in order possibly to define neuropsychological "markers" allowing an early (neuropsychological) diagnosis. We also included a control for some immunological and virological parameters, aiming at better specifying the possible correlations between cognitive and immunological decline.

This paper presents preliminary results in a group of AIDS patients early in the course of the disease and free from any detectable signs of CNS involvement, and a group of asymptomatic HIV seropositive subjects, both 
Table 1 Demographic characteristics for AIDS, asymptomatic HIV seropositive (aHIV) and control (Con) groups. Means (SD). Post hoc comparisons: Duncan test

\begin{tabular}{|c|c|c|c|c|c|c|}
\hline & $\begin{array}{l}A I D S \\
(n=36)\end{array}$ & & $\begin{array}{l}a-H I V \\
(n=33)\end{array}$ & & $\begin{array}{l}\text { Con } \\
(n=26)\end{array}$ & p Value \\
\hline Males/Females & $26 / 10$ & & $21 / 12$ & & $18 / 8$ & ns \\
\hline Age & $\begin{array}{l}33 \cdot 9 \\
(8 \cdot 1)\end{array}$ & & $\begin{array}{l}31 \cdot 0 \\
(7 \cdot 1)\end{array}$ & & $\begin{array}{l}31 \cdot 4 \\
(9 \cdot 5)\end{array}$ & ns \\
\hline Education & $\begin{array}{l}11.2 \\
(3.9)\end{array}$ & & $\begin{array}{l}11 \cdot 3 \\
(4 \cdot 5)\end{array}$ & & $\begin{array}{l}11 \cdot 2 \\
(2.9)\end{array}$ & ns \\
\hline Mini mental state & $\begin{array}{l}28 \cdot 0 \\
(2 \cdot 0)\end{array}$ & & $\begin{array}{l}28 \cdot 6 \\
(1 \cdot 2)\end{array}$ & & $\begin{array}{l}28 \cdot 7 \\
(1 \cdot 3)\end{array}$ & ns \\
\hline Anxiety (Zung scale) & $\begin{array}{l}38.5 \\
(8.1)\end{array}$ & $=$ & $\begin{array}{l}36 \cdot 1 \\
(9 \cdot 8)\end{array}$ & $>$ & $\begin{array}{l}30 \cdot 7 \\
(6.4)\end{array}$ & $<0.01$ \\
\hline Depression (Zung scale) & $\begin{array}{l}38 \cdot 3 \\
(8 \cdot 5)\end{array}$ & $=$ & $\begin{array}{l}37 \cdot 3 \\
(7 \cdot 9)\end{array}$ & $>$ & $\begin{array}{l}31 \cdot 1 \\
(7 \cdot 2)\end{array}$ & $<0.01$ \\
\hline
\end{tabular}

studied in comparison with a group of HIV seronegative controls.

\section{Materials and methods \\ SUBJECTS}

Ninety five subjects (36 AIDS patients, 33 asymptomatic HIV seropositive and $26 \mathrm{HIV}$ seronegative controls) were recruited for this study on the basis of the following criteria: (a) For all subjects. Age between 20 and 40; at least 5 years of schooling; no prior neurological disease or head trauma; no history of drug and/or alcohol abuse; no recent psychotropic medication; no major affective disorder (DSM-III-R criteria); no clinical evidence of dementia or CT scan signs of diffuse brain damage (atrophy). (b) For AIDS patients. Belonging to groups IV $\mathrm{C} 1$ of the CDC classification; no clinical and/or laboratory (blood, CSF, EEG, CT scan) evidence for opportunistic infections or neoplasms of the CNS. (c) For HIV seropositive subjects. Belonging to group II of the CDC classification.

All the subjects examined were outpatients of the department of infectious diseases of the Catholic University of Rome. AIDS patients were 2-6 months from the onset of the symptoms that led to the diagnosis of AIDS. The indicator diseases for AIDS were among the CDC defined AIDS associated secondary infections, involving almost exclusively the digestive and the respiratory tracts (in order of frequency: oesophageal and tracheobronchial candidiasis. Pneumocystis carinii pneumonia, pulmonary candidiasis, mucocutaneous or disseminated herpes simplex, cryptosporidiosis). At the time of neuropsychological assessment, however, all AIDS patients were in fairly good condition and attended the outpatient service for routine follow up. None of them was clinically demented or exhibited any CT scan signs of focal or diffuse brain damage. HIV seropositive subjects were completely asymptomatic, except for immunological abnormalities (CD4 + cell counts less than $7 \times 10^{5} / 1$; $\mathrm{CD} 4+/ \mathrm{CD} 8+$ ratio less than $1 \cdot 0)$. The presumptive dates of seroconversion ranged from 4 to 22 months. Controls were HIV seronegative subjects referred to the outpatient service of the department of infectious diseases for routine controls, after recovery from $\mathrm{HBV}$ hepatitis.
Table 1 reports the demographic characteristics for AIDS patients, HIV seropositive subjects and controls, as well as their mean scores on Mini Mental State ${ }^{18}$ and on Zung scales for anxiety and depression. ${ }^{19} 20$ The three groups did not differ in sex ratio, mean age, or years of schooling. Mini Mental State, administered as a screening measure of the overall cognitive level, did not show significant intergroup differences. Anxiety and depression levels were significantly higher in AIDS and HIV seropositive patients than in controls.

\section{IMMUNOLOGICAL TESTS}

Following recent reports on the most reliable markers of the HIV related immunological decline, ${ }^{21-24}$ we took into account the following cellular and serological parameters: (1) CD4 + (T helper lymphocyte counts); (2) $\mathrm{CD} 4+/ \mathrm{CD} 8+$ ( $\mathrm{T}$ helper / $\mathrm{T}$ suppressor lymphocyte ratio); (3) $\beta$-2-microglobulin; (4) P24 antigen.

The $\mathrm{T}$ lymphocyte subset in peripheral blood was quantified by indirect immunofluorescence using monoclonal antibodies and the percentage of $\mathrm{CD} 4+$ and $\mathrm{CD} 8+\mathrm{T}$ cells was determined taking total and differential white cell count and multiplying for the appropriate factor on flow cytometry.

$\beta$-2-microglobulin was assessed by means of radioimmunoassay procedures (Pharmacia, Uppsala, Sweden).

P-24 antigen was measured by means of a solid phase enzyme immunoassay (Dupont, NEN Research Products, Wilmington, DE, USA). The concentrations in serum samples were interpolated from a standard curve. The reaction was considered as negative (neg) or positive (pos) according to whether the P-24 antigen levels were lower or higher than $0.02 \mathrm{ng} / \mathrm{ml}$.

Table 2 shows results of the immunological tests in AIDS and HIV seropositive subjects. As expected, for both $\mathrm{CD} 4+$ and $\mathrm{CD} 4+/ \mathrm{CD} 8$ + ratio there was a striking difference between AIDS and asymptomatic HIV seropositive patients, owing to lower values found in AIDS. On the other hand, $\beta-2$ microglobulin was significantly higher in AIDS patients than in HIV seropositive subjects, whereas P-24 antigen positivity was equally distributed in the two groups.

Table 2 Immunological data for AIDS and asymptomatic HIV seropositive (a-HIV) groups. Means (SD)

\begin{tabular}{|c|c|c|c|}
\hline & AIDS & $a-H I V$ & p Value \\
\hline $\begin{array}{l}\text { CD4 + cells } \times 10^{6} / 1 \\
\text { (normal: } 762(50))\end{array}$ & $\begin{array}{c}49 \cdot 1 \\
(72 \cdot 5)\end{array}$ & $\begin{array}{c}389 \cdot 5 \\
(238 \cdot 1)\end{array}$ & $<0.001$ \\
\hline $\begin{array}{l}\text { CD4 + CD }+ \text { cells } \times 10^{6} / 1 \\
(\text { normal: } 1 \cdot 7(0 \cdot 9)) \\
\beta-2-\text {-microglobulin } \\
(\text { normal: }<2000 \mathrm{ng} / \mathrm{ml})\end{array}$ & $\begin{array}{c}0 \cdot 1 \\
(0 \cdot 1)\end{array}$ & $\begin{array}{c}0.5 \\
(0 \cdot 2)\end{array}$ & $<0.001$ \\
\hline$<2000$ & $13 / 36$ & $19 / 33$ & \\
\hline $2000-10000$ & $10 / 36$ & $12 / 33$ & $<0.01$ \\
\hline $\begin{array}{c}>10000 \\
P-24 \text { antigen }\end{array}$ & $13 / 36$ & $2 / 33$ & \\
\hline (normal: $<0.02 \mathrm{ng} / \mathrm{ml}$ ) & & & \\
\hline $\begin{array}{l}\text { negative }(<0.02 \mathrm{ng} / \mathrm{ml}) \\
\text { positive }(>0.02 \mathrm{ng} / \mathrm{ml})\end{array}$ & $\begin{array}{l}20 / 36 \\
16 / 36\end{array}$ & $\begin{array}{l}20 / 33 \\
13 / 33\end{array}$ & ns \\
\hline
\end{tabular}


NEUROPSYCHOLOGICAL TESTS

We gave a battery of 17 tests to all subjects (AIDS, HIV seropositive, and controls), providing 22 neuropsychological measures. The 17 tests were subdivided into six groups, roughly corresponding to six different cognitive domains.

Eight of the 17 tests, those marked with an asterisk $\left(^{\star}\right)$ in the list reported below, were taken from the Mental Deterioration Battery, ${ }^{25}$ a set of cognitive tasks devised and currently used in our neuropsychological service. They are not referenced individually and will therefore be described briefly.

(1) Verbal Memory Tasks

Digit span forward and backward. ${ }^{26}$

Rey's auditory verbal learning Test or Rey's '15 mots', ${ }^{27}$ providing three different measures: short term recall, long term recall, and delayed recognition.

(2) Visual and Spatial Memory Tasks Abstract visual memory. ${ }^{\star}$ Twenty two abstract patterns are successively presented; the patient must recognise each pattern, immediately after its presentation, among four similar alternatives.

Figurative visual memory. ${ }^{\star}$ The patient must recognise 10 figures-among visual, semantic, and unrelated alternatives-immediately after two successive presentations and then after a 15 minute interval. The overall correct responses minus the overall false alarms in the two immediate-recognitions constitute the figurative visual memory short term score, whereas the figurative visual memory longterm score is given by the correct responses minus the false alarms in the delayed condition.

Spatial span forward and backward, also known as the Corsi's tapping test. ${ }^{28}$

(3) Verbal Tasks

Semantic verbal fluency. ${ }^{\star}$ The patient is requested to say, within 2 minutes, as many items as possible, belonging to a given semantic category (for example, "flowers," "birds," etc).

Analogies. ${ }^{\star}$ The patient is asked to complete a set of 20 verbally presented analogies (for example, sheep/lamb $=$ cow $\ldots \rightarrow$ "calf").

Phrase construction. ${ }^{\star}$ The patient is requested to produce meaningful and grammatically correct sentences using two or three words (nouns, verbs, prepositions, etc) provided by the examiner.

(4) Visual Spatial Tasks

Copying drawings. The patient must reproduce the models of a star, a cube, and a house.

Copying drawings with landmarks. ${ }^{\star}$ The patient must draw the same items of copying drawings, but using landmarks previously marked on the paper sheet.

Raven's coloured progressive matrices. ${ }^{29}$

(5) "Frontal" Tasks

Phonological verbal fluency. ${ }^{30}$

Wisconsin card sorting. ${ }^{31}$

Temporal rule induction. * Two tokens of different colours (red and blue) are successively presented, following different binary temporal sequences: ' $r-b, r-b, r-b, \ldots$ '. or "rr- bb, rr-bb, rr-bb, ...." or "r-bb, r-bb, r-bb, ...". The criterion is reached when the patient is able to foretell correctly three consecutive sequences.

(6) Time Dependent Tasks

Barrage 1.* In this "line cancellation task", the patient must cross, as quickly as possible, all the lines scattered on a sheet of paper.

Barrage 2. ${ }^{\star}$ The patient is presented with an array of little squares, each containing two variously oriented lines, and requested to mark all the squares identical to a model placed on the top.

Symbol digit substitution of the WAIS. ${ }^{26}$

\section{Results}

Mean scores obtained by AIDS, HIV seropositive, and control subjects on the 22 neuropsychological measures were compared using a series of multivariate and univariate analyses of variance: overall MANOVA, plus separate MANOVAs for groups of tests exploring different cognitive domains, plus individual ANOVAs in order to assess the neuropsychological measures on which the three groups significantly differed. Furthermore, since both anxiety and depression scores, which were significantly higher in AIDS patients and HIV seropositive subjects than in controls, were found to be negatively correlated to most of the neuropsychological test scores, a parallel series of analyses of covariance), overall MANCOVA, plus different MANCOVAs for different cognitive domains, plus individual ANCOVA for the various neuropsychological measures, with anxiety and depression scores as covariates, were also carried out. A type I error rate of 0.01 was used to identify group differences considered to be significant. Results with a probability between 0.05 and 0.01 were considered marginally significant. Post hoc comparisons were performed by means of the Duncan test.

As reported in table 3, both the overall MANOVA and MANCOVA yielded significant $F$ values. Considering the various groups of tests, significant results were obtained by either the MANOVA or the MANCOVA for verbal memory tasks, "frontal" tasks, timedependent tasks. As for verbal tasks, results of the MANOVA were marginally significant, but covarying for anxiety and depression (MANCOVA) led to disapperance of significance. On the other hand, neither the MANOVA nor the MANCOVA yielded significant $F$ values for visual and spatial memory and visuospatial tasks.

A series of univariate analyses of variance (ANOVAs) pointed out significant intergroup differences for Rey's short term and long term recalls, Digit span backward, Spatial span backward, Phrase construction, Wisconsin card sorting, Temporal rule induction, Barrage 1 and 2 and Symbol digit substitution. In all cases, post hoc comparisons (Duncan test) showed that the main source of variance were the poor performance of AIDS patients. HIV seropositive subjects performed 
Table 3 Neuropsychological test performance for AIDS, asymptomatic HIV seropositive (a-HIV), and seronegative control (Con) groups

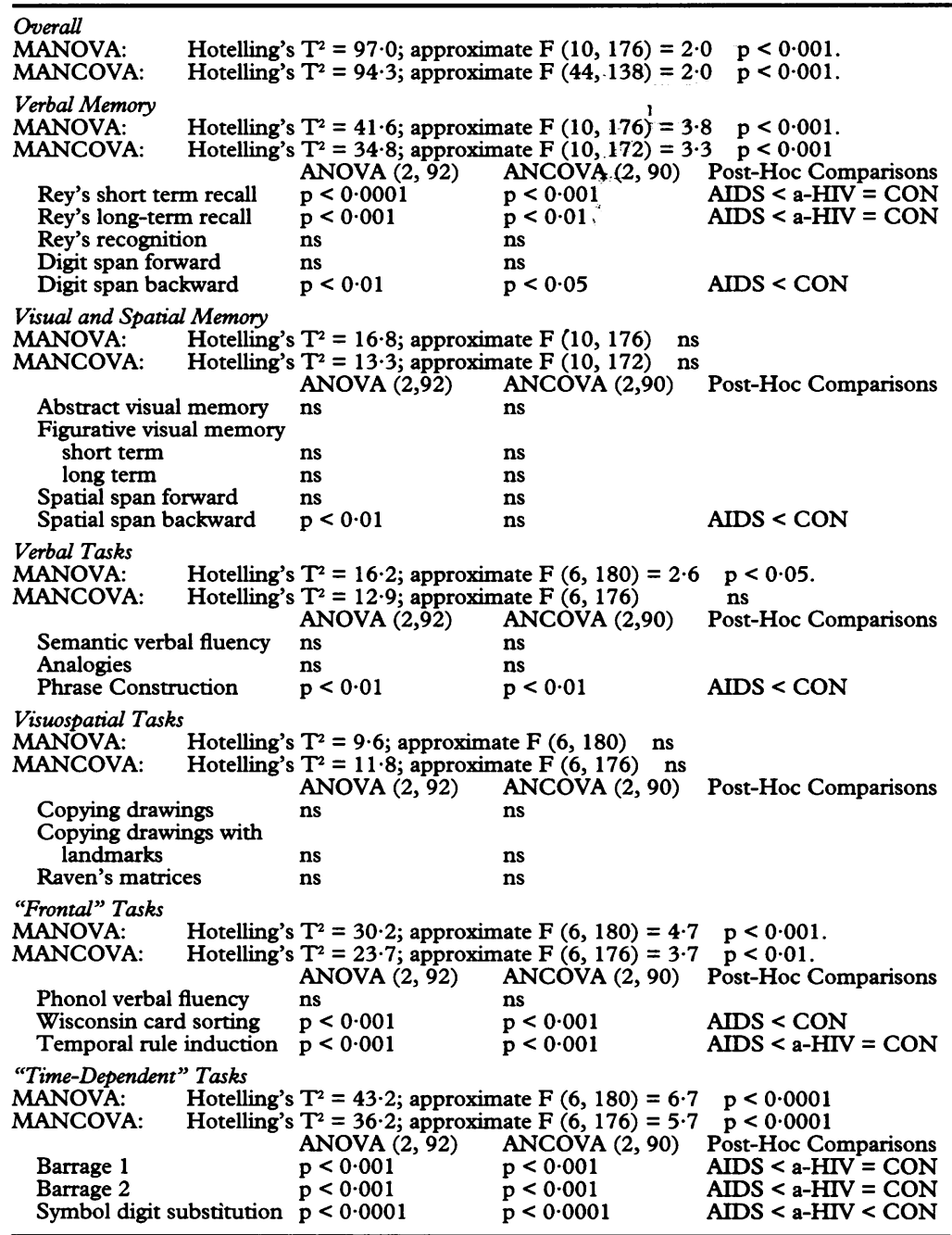

Multivariate analysis of variance (MANOVA) and covariance (MANCOVA) with anxiety and depression as covariates. Post hoc comparisons: Duncan test.

somewhat worse than controls, but only in one case (Symbol digit substitution) did the difference reach the level of statistical significance. Covarying for anxiety and depression scores (ANCOVAs) did not substantially change the results of comparisons among the three groups, except for Spatial span backward, in which the ANCOVA lowered intergroup differences below the level of statistical significance.

Since group means could mask the presence of cognitive impairment within the group of asymptomatic seropositives if only few individuals were so affected, data were also treated in a different way that is, considering the "deviations from the norms". Neuropsychological test scores were corrected for anxiety and depression scores on the basis of a multiple linear regression. Then, since scores obtained by controls on each of the 22 neuropsychological measures were normally distributed, for each of the 22 neuropsychological measures a cut off "pathological" "non-pathological" was established taking the mean score of the controls minus 2 SD. Scores of all the examined subjects on a given neuropsychological measure were then classified as "pathological" or "non- pathological" according to whether they fell below or above the pertinent cut off.

For 25 controls out of $26(96 \cdot 15 \%)$ the number of "pathological" scores ranged from 0 to 2 . Only one control subject $(3.9 \% ; 95 \%$ confidence limits $0 \cdot 1-20 \%$ ) performed pathologically on more than two (namely, on four) tasks. Thus, " 2 " (as overall number of "pathological" scores) was taken as a "cut off" to separate subjects "with" and "without" cognitive impairment. This means that a patient was classified as "impaired" if he/she obtained more than two "pathological" scores and "non-impaired" if he/she did not exceed the number of two "pathological" scores (performing normally on 20 to 22 neuropsychological measurements). In this way, 19/36 AIDS patients $(52 \cdot 8 \% ; 95 \%$ confidence limits: $\quad 35-70 \%$ ) and $10 / 33$ asymptomatic seropositive subjects $(30.3 \% ; 95 \%$ confidence limits: $15-49 \%$ ) could be classified as "impaired". Statistical analysis showed that, in comparison with the controls, there was a significant higher prevalence of subjects "with" cognitive impairment not only among the AIDS patients $\left(\chi^{2}=16.5, \mathrm{p}<0.001\right)$, but even among the asymptomatic seropositive subjects $\left(\chi^{2}=6.7, \mathrm{p}<0.01\right)$.

We also treated the neuropsychological test results by means of a Multidimensional Analysis (MA), a factorial and cluster analysis exploiting a complex set of computerised (mathematical, geometrical, and physical) procedures, ${ }^{32}$ in order to see whether the examined subjects could be blindly grouped into classes, corresponding to different levels of overall cognitive performance. The MA showed that a partition in two classes was the best possible one, since it ensured the maximum of homogeneity "within" and the maximum of difference "between" classes, in terms of neuropsychological test scores. The two classes, reflecting two distinct levels of overall cognitive functioning (lower level = "impaired" higher level = "non-impaired"), were constituted by 32 and 63 subjects, respectively. The distribution of "impaired" and "non-impaired" subjects across the three original experimental groups was similar to that previously obtained, on the basis of the cut off number of pathological neuropsychological performances: "impaired" subjects were 20/36 AIDS patients (55.6\%), 10/33 asymptomatic seropositive subjects $(30 \cdot 3 \%)$, and $2 / 26$ controls $(7 \cdot 7 \%)$. Comparisons between either AIDS patients or asymptomatic seropositive subjects and controls yielded significant results $\left(\chi^{2}=15 \cdot 1, \mathrm{p}<0.001\right.$ and $\chi^{2}=5.6, \mathrm{p}<0.01$, respectively).

As for the cellular and serological markers, comparisons between "impaired" and "nonimpaired" subjects, performed in order to find out significant relationships between levels of cognitive functioning and immunological status, yielded the following results: (a) Considering the overall groups of "impaired" and "non-impaired" (either AIDS or asymptomatic seropositive) subjects, low values of $\mathrm{CD} 4+$ and $\mathrm{CD} 4+/ \mathrm{CD} 8+$ and high titres of $\mathrm{P}-24$ antigen tended to prevail among 
"impaired" subjects (Mann-Whitney U Test significant at $\mathrm{p}<0.05$ for either CD4 + or CD4 + $/ \mathrm{CD} 8+; \chi^{2}$ significant at $\mathrm{p}<0.05$ for P-24 antigen); however, $\beta$-2-microglobulin levels were not different in the two groups, (b) Within the AIDS group there was no significant difference between "impaired" and "non-impaired" subjects in relation to CD4+/CD8 +, $\beta$-2-microglobulin, P-24 antigen. (c) Within the asymptomatic seropositive group, $\mathrm{CD} 4+$ and $\mathrm{CD} 4+/ \mathrm{CD} 8+$ were lower in "impaired" than in "non-impaired" subjects (Mann-Whitney U Test significant at $\mathrm{p}<0.05$ for $\mathrm{CD} 4+$ and at $\mathrm{p}<0.01$ for $\mathrm{CD} 4+/ \mathrm{CD} 8+) ; \quad \beta$-2-microglobulin levels were homogeneous in the two groups; $\mathrm{P}-24$ antigen positivity was prevalent in "impaired" subjects $\left(\chi^{2}\right.$ with Yates' correction significant at $\mathrm{p}<0.01)$.

\section{Discussion}

Our study shows that patients with AIDS, though clinically non-demented and free from neurological signs or symptoms, nevertheless show a high incidence of cerebral involvement, as reflected by their poor performance on several neuropsychological tasks.

Most sensitive to AIDS cognitive impairment are timed psychomotor tasks (Symbol digit substitution, Barrage 1 and 2): tasks involving sequencing and "set-shifting" (Temporal rule induction, Wisconsin card sorting). Among verbal tasks, only Phrase construction, a high-level verbal task that also implies "abstraction" capacities, is significantly impaired in AIDS patients. As for memory tasks, AIDS patients have difficulties with the Rey's ' 15 mots', particularly when attention, learning, and active retrieval of information are needed (Rey's short term and long term recalls). On the contrary, their performance on recognition memory tasks, either verbal (Rey's delayed-recognition) or visual (Abstract visual memory, Figurative visual memory short term and long term), are relatively good. Analogously, the selective impairment on Digit span backward suggests damage to the more "active" mechanisms of short term memory, with relative sparing of the more "passive" (phonological) routes, presumably involved in digit span forward.

These findings of psychomotor slowing, decreased attention, and poor monitoring of information, for problem solving, learning, and recall, with relatively preserved intelligence (inferential reasoning on either visuospatial or verbal stimuli, as tested by Raven's coloured progressive matrices and by Analogies, respectively), language (as tested by Semantic verbal fluency and Phrase construction), visuoconstructive abilities (Copying drawings, Copying drawings with landmarks), are consistent with a "subcortical" pattern of cognitive impairment". ${ }^{33}$ They confirm results reported in other studies, ${ }^{3} 101216$ though showing a wider range of cognitive tasks on which AIDS patients perform pathologically, and provide further support to the often reported claim that a high risk of cognitive decline develops with the onset of HIV related symptoms or AIDS itself. The cognitive impairment found in our AIDS patients was not attributable to factors such as CNS opportunistic infections or neoplasms, alcohol or drug abuse/addiction, psychiatric status or history (since all these conditions had been excluded in the recruitment of patients), nor was a function of affective changes such as anxiety and depression (neither the ANCOVA nor the regression of neuropsychological test scores against anxiety and depression scores substantially modified the pathological level of AIDS performance). Thus, the neuropsychological impairment found in our AIDS patients could not but represent a specific manifestation of HIV related cerebral damage.

While it is generally accepted by now that a significant cognitive impairment frequently occurs during AIDS, the presence of cognitive abnormalities during early HIV infection is much more controversial, since it has been claimed by several ${ }^{3-10}$ but not all studies. ${ }^{11-14}$ Our asymptomatic HIV seropositives subjects as a group, generally performed somewhat poorer than controls, but only in one case (Symbol digit substitution) was the difference statistically significant. Thus, the hypothesis of an incipient cognitive impairment in asymptomatic seropositive subjects is not confirmed by a cross sectional approach in which groups are compared by measures of central tendency, such as means and standard deviations. However, if one considers the deviations from the norms and the consequent classification of each subject as cognitively "impaired" or "non-impaired" (on the ground of his own composite performance on all the neuropsychological tests), it clearly appears that a significant number of subjects classified as "impaired" fall not only in the AIDS but also in the asymptomatic seropositive group (table 4 ). This means that while many asymptomatic HIV seropositive subjects performed as well as controls on most neuropsychological tests, there was a subsample that clearly performed less well. A discriminant analysis, performed on the whole set of the neuropsychological scores, showed that Symbol digit substitution and Rey's long term recall were the variables with the maximum load in discriminating between "impaired" and "non-impaired" individuals. Thus, in seeking neuropsychological markers for early HIV related cognitive decline, particular attention should be paid to tasks that assess timed psychomotor abilities and to memory tasks that require learning and active retrieval of information.

The discovery, among the asymptomatic seropositives, of a subsample of subjects with subtle but significant cognitive impairment represents one of the most remarkable aspects of our results. It is in agreement with several previous reports ${ }^{3-10}$ : with some of them ${ }^{810}$ even in regard to the pattern of cognitive abilities found to be affected. We found a $30.3 \%$ (95\% confidence limits: 15-49) frequency of cognitive abnormalities in our asymptomatic 
seropositive subjects. Except for the early paper by Grant et $a^{3}$ that claimed a $44 \%$ frequency, estimates of cognitive abnormalities in asymptomatic seropositive subjects reported by the above cited studies are quite similar to ours. On the other hand, frequencies reported by studies, ${ }^{11-14}$ which claimed the absence of significant cognitive changes in asymptomatic seropositive subjects, are obviously lower, ranging from 5 to $20 \%$. Such a discrepancy could be due to methodological variations at different levels (subject recruitment criteria, neuropsychological test batteries, analysis of data, and inferences from results, confidence limits considered), as recently discussed by Stern $e t a l^{9}$ and Lunn $e t$ $a l .{ }^{10}$

As for the immunological parameters, our data sustain the existence of a significant relationship between cognitive and immunological decline, at least in early stages of HIV infection. In the asymptomatic seropositive, but not in the AIDS, group, subjects "with" cognitive impairment had $\mathrm{CD} 4+$ and $\mathrm{CD} 4+/ \mathrm{CD} 8+$ significantly lower than subjects "without" cognitive impairment. On the basis of these findings, it could be hypothesised that, in asymptomatic seropositive subjects, HIV primes damage to CNS (eventually resulting in cognitive symptoms) coinciding with an immunological deficit, signalled by CD4 + lymphocyte decrease. The lack of a significant correlation between cognitive impairment and high serum levels of $\beta-2$ microglobulin, seems to deny, in either AIDS or asymptomatic seropositives, the participation of an immune activation in the development of HIV related CNS damage. It is particularly worth noting the coincident occurrence of high titres of P-24 antigen and of cognitive abnormalities that we found again in asymptomatic seropositive subjects. A persistent viral production with CNS stable infection could be the possible mechanism underlying this phenomenon. On the grounds of a significant immunological decline (indicated by low $\mathrm{CD} 4+$ and $\mathrm{CD} 4+/ \mathrm{CD} 8+$ ) a high rate of viral replication is probably involved in either onset or progression of neuropsychological symptoms. If the immunological breakdown underlies the evolution of HIV infection from the phase of latency to that of active replication, the increased viral replication, in turn, is likely to induce an upregulation of cytokine (TNF $a$, IL-6, $\gamma$ IFN) release, priming a sort of chain reaction. The subsequent steps would be: amplification of HIV infection with high levels of viral production; active expression in CNS macrophages; infection of several cell types, block of neurotransmission by HIV envelope proteins, and, finally, cellular damage mediated by increased cytokine production. ${ }^{34-36}$

The cognitive abnormalities which we found were consistent enough as to define a neuropsychological syndrome that should be recognised as one of the early manifestations of the direct effect of HIV on CNS. Longitudinal studies will presumably clear whether the present cross sectional findings of cognitive abnormalities in HIV-infected individuals are predictive of progressive cognitive decline-that is, whether those individuals at present classified as "impaired" will develop a proper ADC.

1 Hollander H, Levy JA. Neurologic abnormalities and recovery of human immunodeficiency virus from cererecovery of human immunodeficiency virus from

2 Navia BA, Jordan BD, Price RW. The AIDS dementia complex. I. Clinical features. Ann Neurol 1986;19: 517-24.

3 Grant I, Atkinson JH, Hesselink JR, et al. Evidence for early central nervous system involvement in the acquired immunodeficiency syndrome (AIDS) and other human immunodeficiency virus (HIV) infections. Ann Intern Med 1987;107:828-36.

4 Saykin AJ, Janssen RS, Sprehn GC, et al. Neuropsychological dysfunction in AIDS-related complex. Neurology 1987;37(suppl):374.

5 Saykin AJ, Janssen RS, Sprehn GC, et al. Neuropsychological dysfunction in HIV-infection: characterization in a lymphoadenopathy cohort. Int $\mathcal{f}$ Clin zeuropsychol 1988;10:81-95.

6 Field M, Tate J, Kunze H, et al. Cerebral dysfunction with evidence of cerebral HIV infection among Asymptomatic HIV Seropositive subjects. Aust NZ $\mathcal{f}$ Med 1989;19:694-9.

7 Claypoole KHG, Townes BD, White D, et al. Neuropsychological aspects of early HIV infection. $\dot{f}$ Clin Exp Neuropsychol 1990;12:17.

8 Wilkie FL, Eisdorfer C, Morgan R, et al. Cognition in early human immunodeficiency virus infection. Arch Neurol 1990;47:433-40.

9 Stern Y, Marder K, Bell K, et al. Multidisciplinary baseline assessment of homosexual men with and without human immunodeficiency virus infection. III. Neurologic and neuropsychological findings. Arch Gen Neurologic and neuropsych
Psychiatry 1991;48:131-8.

10 Lunn S, Skydsbierg M, Schulsinger $H$, et al. A preliminary report on the neuropsychologic sequelae of human immunodeficiency virus. Arch Gen Psychiatry 1991;48: $139-42$.

11 Goethe KE, Mitchell JE, Marshall DW, et al. Neuropsychological and neurological function of human immunodeficiency virus seropositive asymptomatic individuals. Arch Neurol 1989;46:129-33.

12 Miller EN, Selnes OA, McArthur MB, et al. Neuropsychological performance in HIV-1-infected homosexual men: the Multicenter AIDS Cohort Study (MACS). Neurology 1990;40:197-203.

13 Franzblau A, Letz R, Hershman D, et al. Quantitative neurologic and neurobehavioral testing of persons infected with human immunodeficiency virus type 1 . infected with human imm

14 Selnes OA, Miller E, McArthur MB, et al. HIV-1 infection: no evidence of cognitive decline during asymptomatic stages. Neurology 1990;40:204-8.

15 Sidtis JJ, Tross S, Navia BA, et al. Neuropsychological characterization of the AIDS dementia complex. In: Proceedings of the International Conference on AIDS, Paris, France, June 23-25, 1986 (abstract).

16 Tross S, Price RW, Navia BA, et al. Neuropsychological characterization of the AIDS dementia complex: a preliminary report. AIDS 1988;2:81-8.

17 Van Gorp WG, Mitrushina M, Cummings $\mathrm{L}$, et al. Normal aging and the subcortical encephalopathy of AIDS: a neuropsychological comparison. Neuropsychiat Neuropsychol Behav Neurol 1989;2:5-20.

18 Folstein MF. The Mini-Mental State Examination. In: T Cook, S Ferris, R Bartus, eds, Assessment in geriatric psyCook, S Ferris, R Bartus, eds, Assessment in geriatric psy-
chopharmacology New Canaan, CT. Mark Powley chopharmacology

19 Zung WWK. Self-rating depression scale in an outpatient clinic. Arch Gen Psychiat 1965;13:508-15.

20 Zung WWK. A rating instrument for anxiety disorders. Psychosom 1971;12:371-9.

21 Fahey JL, Taylor JMG, Detels R, et al. The prognostic value of cellular and serologic markers in infection with human immunodeficiency virus type $1 . N \mathrm{Engl} f \mathrm{Med}$ 1990;322:166-72.

22 Phillips AN, Lee CA, Elford J, et al. Serial CD4 lymphocyte counts and development of AIDS. Lancet 1991; 337:389-92.

23 Lacey JN, Forbes MA, Waugh MA, et al. Serum beta-2microglobulin and human immunodeficiency virus infection. AIDS 1987;1:123-7.

24 De Wolf F, Goudsmit J, Paul DA, et al. Risk of AIDS related complex and AIDS in homosexual men with persistent HIV antigenaemia. BMF 1987;295:569-72.

25 Caltagirone C, Gainotti G, Masullo C, et al. Validity of some neuropsychological tests in the assessment of some neuropsychological tests in the assessment of
mental deterioration. Acta Psychiat Scandinav 1979;60: menta.

26 Wechsler D. Wechsler adult intelligence scale-revised. New York: The Psychological Corporation, 1981.

27 Rey A. Memorisation d'une serie de 15 mots en 5 repeti- 
tions. In: A. Rey, ed, L'examen clinique en psychologie Paris: Presses Universitaries, 1958.

28 Corsi PM. Human memory and the medial temporal regions of the brain, $\mathrm{Ph} \mathrm{D}$ thesis, McGill University, 1972.

29 Raven JC. Progressive Matrices, Sets $A, A b . B$ London: Raven JC. Progressive Matrices, Sets $A$,

30 Borkowsky JG, Benton AI, Spreen O. Word fluency and Borkowsky JG, Benton AI, Spreen O. Word fluenc
brain damage. Neuropsychologia 1967;5:135-40.

31 Heaton R K. Wisconsin card sorting test manual. Odessa Psychological Assessment Resources inc, 1981

32 Costa P, Maravalle M, Salani MP, et al. 'introduzione all'analisi dei dati multidimensionali'. Rome, Università 'La Sapienza', 1981.
33 Cummings JL, Benson DF. Subcortical dementia-review of an emerging concept. Arch Neurol 1984;41:874-9.

34 Fauci AS, Schmittman SM, Poli G, et al. Immunopathogenetic mechanisms in Human Immunodeficiency pathogenetic mechanisms in Human Immunodeficiency 678-93.

35 Fauci AS. The human immunodeficiency virus infectivity and mechanisms of pathogenesis. Science 1988;239: 617-22.

36 Gallo P, Frei K, Rordorf C, et al. Human immunodeficiency virus type 1 (HIV-1) infection of the CNS: an evaluation of cytokines in cerebrospinal fluid. $\mathcal{F}$ Neuroimmunol 1989;23:109-16. 